\title{
School teachers profit by observing school children's basic life support training
}

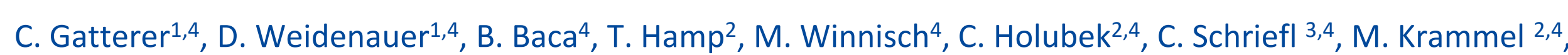
F. Sterz ${ }^{2,4}$, H. Herkner ${ }^{2,4}$, H. Domanovits ${ }^{2,4}$

Contact: gatterer@puls.at

\section{Purpose}

As part of a project to improve the rate of bystander CPR, 3rd grade primary school children were trained in CPR and using an AED. We investigated the effect CPR trainings of school classes had on the teacher, who was observing the training. The aim was to evaluate previous knowledge and its improvement. Additionally changes in self-confidence of teachers to provide bystander CPR after observing the training were evaluated.

\section{Methods}

During the CPR training for pupils, their teachers were present. We asked primary school teachers to participate in a written survey regarding to the training of the pupils. The participants had to complete two surveys, one before and another one after the training. In total 197 teachers participated in our survey.
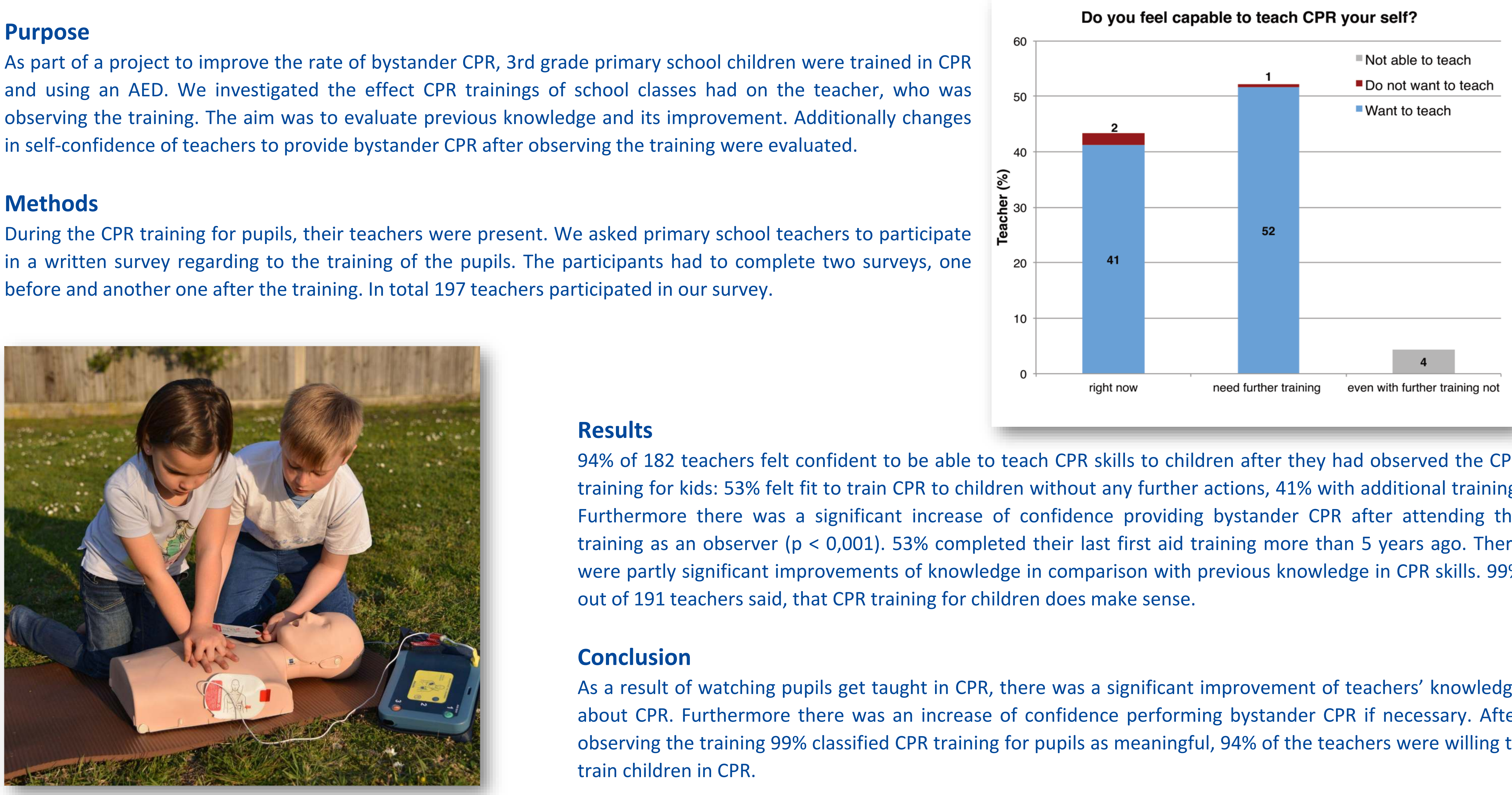

\section{Results}

94\% of 182 teachers felt confident to be able to teach CPR skills to children after they had observed the CPR training for kids: $53 \%$ felt fit to train CPR to children without any further actions, $41 \%$ with additional training. Furthermore there was a significant increase of confidence providing bystander CPR after attending the training as an observer $(p<0,001) .53 \%$ completed their last first aid training more than 5 years ago. There were partly significant improvements of knowledge in comparison with previous knowledge in CPR skills. 99\% out of 191 teachers said, that CPR training for children does make sense.

\section{Conclusion}

As a result of watching pupils get taught in CPR, there was a significant improvement of teachers' knowledge about CPR. Furthermore there was an increase of confidence performing bystander CPR if necessary. After observing the training $99 \%$ classified CPR training for pupils as meaningful, $94 \%$ of the teachers were willing to train children in CPR.

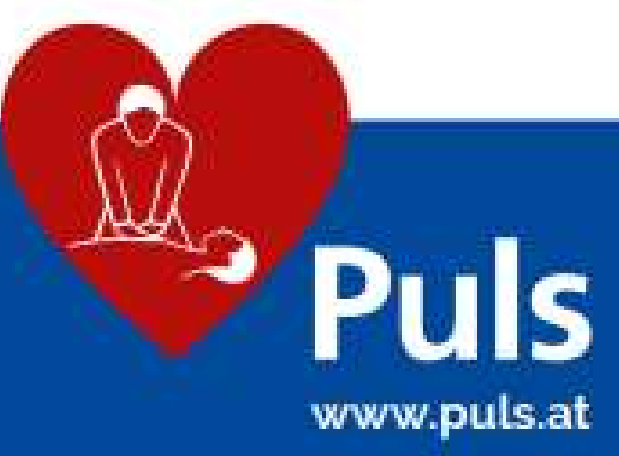

